Catastrophizing Scale (PCS, 0-52 scale) at the baseline examination in 20162017. Bilateral interphalangeal, metacarpophalangeal, first carpometacarpal and the scaphotrapeziotrapezoidal joint were scored for radiographic OA according to the Kellgren-Lawrence (KL) index (sum score: 0-132 scale). Using linear regression analyses, we analysed whether KL sum score, HADS sum score and PCS sum score (independent variables) were associated with AUSCAN pain (dependent variable). Separate models were applied for each independent variable with adjustment for age, sex and body mass index (BMI). Thereafter, all independent variables were included in the same model. Analyses were repeated using NRS hand pain as the dependent variable. Interactions between $\mathrm{KL}$ sum score and HADS/PCS were explored.

Results: Patients reported wide range of pain severity with mean (SD) AUSCAN pain of 8.2 (4.0) and mean (SD) NRS hand pain of 3.8 (2.3). Their radiographic severity ranged from minimal to severe with a median (IQR) KL sum score of 28 (15-44). Most patients reported low levels of anxiety, depression and pain catastrophizing with median (IQR) HADS sum score of 6 (3-10) and PCS sum score of $9(5-15)$.

The HADS and PCS sum scores were associated with hand pain, both when analysed separately and in the same model together with $\mathrm{KL}$ sum score (table 1). Increasing radiographic severity was not associated with hand pain in the age, sex and BMI-adjusted models. When including HADS and PCS in the models, the associations between radiographic severity and pain became stronger for both pain outcomes and statistically significant for AUSCAN pain (table 1).

We found interactions between KL sum score and HADS. In the 61 persons with HADS depression and anxiety subscale scores below 8 , the $\mathrm{KL}$ sum score was significantly associated with AUSCAN pain $(B=0.03,95 \% \mathrm{Cl}: 0.003$ to 0.06 , $\mathrm{p}=0.03$ ) and NRS pain ( $\mathrm{B}=0.02,95 \% \mathrm{Cl}: 0.002$ to $0.03, \mathrm{p}=0.03$ ) (adjusted for age, sex, BMI and PCS), whereas no associations between radiographic severity and pain was found in persons with HAD depression and/or anxiety subscale scores of 8 or more.

Abstract OP0054 - Table 1

\begin{tabular}{|l|l|l|l|l|}
\hline & \multicolumn{2}{|l|}{ AUSCAN pain $(\mathrm{B}, 95 \% \mathrm{Cl})$} & \multicolumn{2}{l|}{ NRS hand pain $(\mathrm{B}, 95 \% \mathrm{Cl})$} \\
\hline & Model $1^{*}$ & Model $2 * *$ & Model $1^{*}$ & Model $2^{* *}$ \\
\hline KL sum score & $0.01(-0.01,0.04)$, & $0.03(0.001,0.05)$, & $0.005(-0.01,0.02) ;$ & $0.01(-0.002,0.03) ;$ \\
& $p=0.35$ & $p=0.04$ & $p=0.52$ & $p=0.09$ \\
\hline HADS sum & $0.19(0.11,0.26) ;$ & $0.12(0.04,0.20) ;$ & $0.11(0.07,0.15) ;$ & $0.08(0.03,0.12) ;$ \\
score & $p<0.001$ & $p=0.01$ & $p<0.001$ & $p=0.001$ \\
\hline PCS sum score & $0.17(0.12,0.23) ;$ & $0.14(0.08,0.20) ;$ & $0.10(0.07,0.13) ;$ & $0.07(0.04,0.11) ;$ \\
& $p<0.001$ & $p<0.001$ & $p<0.001$ & $p<0.001$ \\
\hline
\end{tabular}

HADS and PCS isclud KL, HADS and PCS, respectively, with adj. for age, sex

Conclusions: Depression, anxiety and pain catastrophizing were associated with pain in hand OA, emphasising that pain in hand OA should be treated in a biopsychosocial framework. Importantly, radiographic severity was associated with pain only in persons with no or low levels of depression and anxiety.

Disclosure of Interest: None declared

DOI: 10.1136/annrheumdis-2018-eular.1939

\section{OP0055 A NOVEL METHOD FOR ASSESSING PROXIMAL TIBIOFIBULAR JOINT ON MR IMAGES IN PATIENTS WITH KNEE OSTEOARTHRITIS}

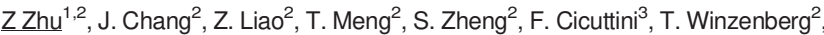
A. Wluka ${ }^{3}$, D. Jiang ${ }^{4}$, W. Han ${ }^{2}$, C. Ding ${ }^{1,2} .{ }^{1}$ Clinical Research Center, Zhujiang Hospital, Guangzhou, China; ${ }^{2}$ Menzies Institute for Medical Research, University of Tasmania, Hobart, ${ }^{3}$ Department of Epidemiology and Preventive Medicine, Monash University, Melbourne; ${ }^{4}$ School of Engineering and ICT, University of Tasmania, Hobart, Australia

Background: Proximal tibiofibular joint (ProxTibFibJ) is a synovial sliding joint that has been estimated to transmit one-sixth of the leg's static load. One study has reported that proximal fibular osteotomy could significantly improve the clinical outcomes in patients with medial compartment OA. However, no study has delineated the measurement of ProxTibFibJ morphological parameters (ProxTibFibJ contacting area, load-bearing area, lateral stress-bolstering area and posterior stress-bolstering area) on magnetic resonance imaging (MRI) and investigated their correlations with knee OA structural abnormalities.

Objectives: To validate a pragmatic method to measure the morphological parameters of the ProxTibFibJ and to describe their associations with knee structural abnormalities in patients with knee osteoarthritis $(\mathrm{OA})$.

Methods: A total of 408 participants with knee OA were selected. The morpholog ical status of ProxTibFibJ were measured on coronal and sagittal magnetic resonance images (MRI). We calculated the contacting area of ProxTibFibJ (S), and its projection areas onto the horizontal (load-bearing area, $\mathrm{S} \tau$ ), sagittal (lateral stress-bolstering area, $S \varphi$ ) and coronal plane (posterior stress-bolstering area, $\mathrm{S} v)$, respectively. Knee structural abnormalities including cartilage defects, bone marrow lesions (BMLs) and cartilage volume were evaluated. Clinical construct validity was examined through describing the associations between the morphological parameters of ProxTibFibJ and knee structural abnormalities. The reliabilities were examined by calculating the intra- and inter-observer correlation coefficients.

Results: The average ProxTibFibJ fibular contacting area was $2.4 \pm 0.7 \mathrm{~cm}^{2}$. The intra- and inter-observer correlation coefficients for all measures were excellent (all $\geq 0.90$ ). In cross-sectional analyses, the ProxTibFibJ morphological parameters $(\mathrm{S}, \mathrm{S} \tau, \mathrm{S} v$ and $\mathrm{S} \varphi)$ were significantly associated with radiographic medial JSN (OR 1.72 for $S ; 2.20$ for $\mathrm{S} \tau ; 1.65$ for $\mathrm{S} v$ ), radiographic medial osteophyte (OR 0.51 for $\mathrm{S} \varphi$ ) and $\mathrm{MRI}$-assessed knee joint structural abnormalities including cartilage volume ( $\beta-0.07$ for $\mathrm{S} ;-0.09$ for $\mathrm{S} \tau$ ), cartilage defects (OR 1.63 for $\mathrm{S} ; 1.95$ for $\mathrm{S} \tau$ ) and BMLs (OR 1.54 for $\mathrm{S} ; 1.74$ for $\mathrm{S} \tau$ ) at medial tibiofemoral compartment. In longitudinal analyses, $S(R R, 1.45)$ and $S \tau(R R, 1.55)$ of ProxTibFibJ were significantly and positively associated with an increase in medial tibial cartilage defects over 2 years, after adjustment for age, gender, height, weight, ROA, tibia plateau bone area and intervention. $\mathrm{S} \tau(\beta,-0.07), \mathrm{S} v(\beta,-0.07)$ and $\mathrm{S}(\beta,-0.06)$ of ProxTibFibJ were significantly and negatively associated with change in medial tibial cartilage volume, after adjusted for above covariates. S $\tau(R R, 1.55)$ of ProxTibFibJ was positively associated with an increase in medial tibial BML, and S (RR, 0.35) was negatively associated with an increase in medial femoral BMLs.
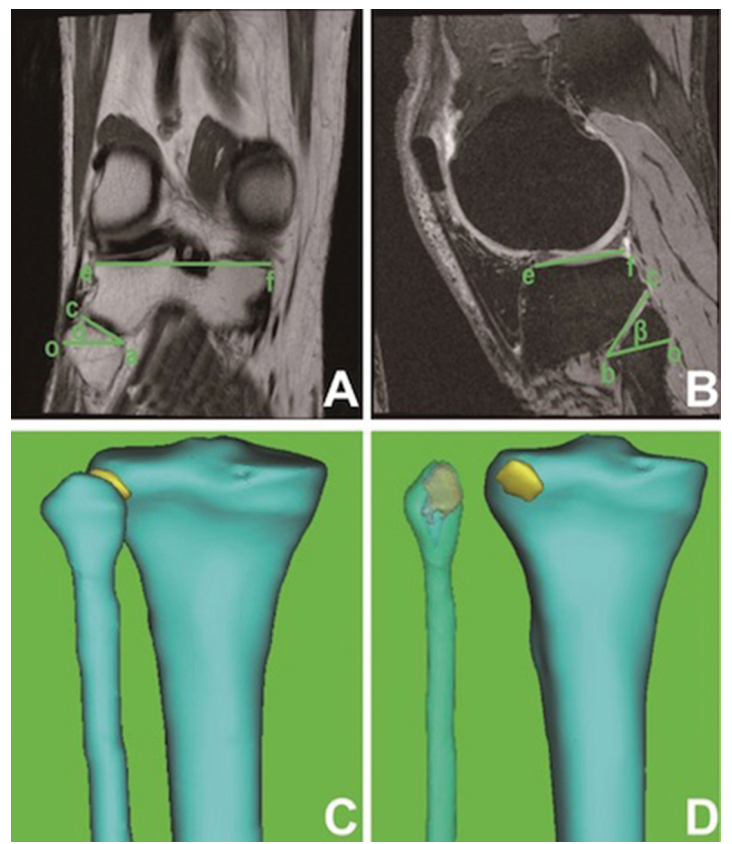

Conclusions: This novel method to assess the morphological parameters of ProxTibFibJ using MRI is reproducible, and has clinical construct validity. The longitudinal associations with osteoarthritic changes suggest that higher load-bearing area of ProxTibFibJ is a potential risk factor for medial compartment OA.

Disclosure of Interest: None declared

DOI: 10.1136/annrheumdis-2018-eular.1685

\section{OP0056 MOLECULAR AND STRUCTURAL BIOMARKERS OF INFLAMMATION AT 2 YEARS AFTER ACUTE ACL INJURY DO NOT PREDICT STRUCTURAL KNEE OSTEOARTHRITIS AT 5 YEARS}

F Roemer ${ }^{1,2,3}$, M. Englund $^{4}$, A. Turkiewicz ${ }^{4}$, A. Struglics ${ }^{1}$, A. Guermazi ${ }^{2}$, L. S. Lohmander ${ }^{1}$, S. Larsson ${ }^{1}$, R. Frobell ${ }^{1}{ }^{1}$ Orthopaedics, Department of Clinical Sciences Lund, Lund University, Lund, Sweden; ${ }^{2}$ Radiology, Boston University School of Medicine, Boston, USA; ${ }^{3}$ Radiology, University of Erlangen-Nuremberg, Erlangen, Germany, ${ }^{4}$ Clinical Epidemiology Unit, Orthopaedics, Department of Clinical Sciences Lund, Lund University, Lund, Sweden

Background: Trauma-induced cytokine response and local inflammation after knee injury may be important in the development of posttraumatic osteoarthritis $(\mathrm{OA})$. It has been reported that synovial fluid levels of inflammatory markers remain increased up to 5 years after anterior cruciate ligament $(A C L)$ injury, indicative of extended local inflammation in the injured joint. ${ }^{1}$ Inflammation potentially represents a valid target for treatment in the early and subacute phase after joint trauma in order to prevent or delay onset of post-traumatic knee OA. 
Objectives: To determine the role of inflammatory biomarkers at 2 years after anterior cruciate ligament $(A C L)$ injury for predicting radiographic and magnetic resonance imaging (MRI)-defined knee OA 5 years post injury. Secondary aim was to estimate the concordance of inflammatory biomarkers assessed by MRI and in synovial fluid.

Methods: We studied 113 patients with acute ACL injury. 1.5 Tesla knee MRIs were read for Hoffa- and effusion-synovitis. Biomarkers of inflammation included IL-6, IL-8, IL-10, TNF- $\alpha$ and IFN- $\gamma$ in serum and synovial fluid, and IL-12p70 in serum. The outcome was radiographic knee OA (ROA) or MRI-defined OA (MROA) at 5 years. Area under receiver operating characteristic curve (AUC), sensitivity and specificity were evaluated in models including MRI features only (M1), inflammation biomarkers only (serum [M2a] - synovial [M2b]) or both MRI and serum [M3a] or synovial [M3b] markers. Linear regression was used for evaluating association between MRI and synovial biomarkers.

Results: At 5 years, ROA was present in $26 \%$ and MROA was present in $32 \%$ of patients' injured knee. The AUCs $(95 \% \mathrm{Cl})$ for ROA were $0.44(0.42-0.47 ; \mathrm{M} 1)$, $0.62(0.59-0.65 ; \mathrm{M} 2 \mathrm{a}), 0.58(0.55-0.61 ; \mathrm{M} 2 \mathrm{~b}), 0.53(0.50-0.56 ; \mathrm{M} 3 \mathrm{a})$ and 0.50 (0.46-0.53;M3b) for each model. The corresponding AUCs for MROA were 0.67 (0.64-0.70), 0.49 (0.47-0.52), $0.65(0.61-0.68), 0.56(0.52-0.59)$ and 0.69 (0.660.72 ) (table 1). The associations between MRI and synovial biomarkers were weak and not statistically significant, apart from effusion-synovitis and IL-8 (log 10 IL8 levels were 0.23 and 0.43 higher in persons with grade 1 or $2 / 3$, respectively).

Abstract OP0056 - Table 1 Discriminatory accuracy of imaging and serum/synovial fluid biomarkers at 2 years with respect to knee osteoarthritis (OA) development at 5 year according to 4 definitions using logistic regression model maximising area under receive operating characteristic curve (AUC)

\begin{tabular}{|c|c|c|c|c|c|c|}
\hline \multicolumn{2}{|l|}{$\begin{array}{l}\text { Outcomo at } 5 \\
\text { yeors }\end{array}$} & $\begin{array}{l}\text { MRI f footures only } \\
\text { (Model 1) }\end{array}$ & $\begin{array}{l}\text { Serum biomorkers } \\
\text { only } \\
\text { (Model 2a) }\end{array}$ & $\begin{array}{l}\text { MRi and Serum } \\
\text { biomontreers } \\
\text { (Model 3a) }\end{array}$ & $\begin{array}{l}\text { Shnovial } \\
\text { biomankrears only } \\
\text { (Model 2b) }\end{array}$ & 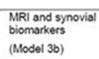 \\
\hline \multirow[t]{3}{*}{ MRI OA } & AUC & $0.67(0.04 \cdot 070)$ & & $0.65(0.61-068)$ & $058(0520.59)$ & 0691068.0727 \\
\hline & Sens & $0.03(0.01: 0.05)$ & $0.00(0.000: 0.00)$ & $0.00(0.000: 0.00)$ & $0.02(0.000 .0 .03)$ & $0.02(0.0600 .0 .03)$ \\
\hline & spoc & $0.98(0.97 ; 1.00)$ & $1.00(1.00 ; 1.00)$ & $1.00(0.99 ; 1.00)$ & $0.98(0.96: 1.00)$ & $0.99(0.88 ; 1.00)$ \\
\hline \multirow[t]{3}{*}{ ROA } & AUC & & & & & \\
\hline & Sons & $\begin{array}{l}0.44(0.42: 0.47) \\
0.00(0.00 ; 0.00)\end{array}$ & $\begin{array}{l}0.62(0.59: 0.65) \\
0.01(0.00 ; 0.03)\end{array}$ & $\begin{array}{l}0.58(0.55: 0.61) \\
0.01(0.00 ; 0.02)\end{array}$ & $\begin{array}{l}0.53(0.50 ; 0.56) \\
0.00(0.00 ; 0.00)\end{array}$ & $\begin{array}{l}0.50(0.46 ; 0.53) \\
0.00(0.00 ; 0.00)\end{array}$ \\
\hline & Spec & $1.00(1.00: 1.00)$ & $1.00(1.00 ; 1.00)$ & $1.00(1.00 ; 1.00)$ & $1.00(1.00 ; 1.00)$ & $1.00(1.00: 1.00)$ \\
\hline \multirow{4}{*}{ TF OA } & AUC & & & & & \\
\hline & & $0.68(0.65 ; 0.71)$ & $0.52(0.50 ; 0.54)$ & $0.64(0.62 ; 0.67)$ & $0.53(0.49,0.56)$ & $0.64(0.60 ; 0.68)$ \\
\hline & Sens & $0.22(0.18 ; 0.26)$ & $0.00(0.00 ; 0.01)$ & $0.13(0.10 ; 0.17)$ & $0.02(0.00 ; 0.04)$ & $0.14(0.10 ; 0.19)$ \\
\hline & Spoce & $0.89(0.86 ; 0.93)$ & $0.99(0.98 ; 1.00)$ & $0.91(0.88: 0.95)$ & $0.95(0.92 ; 0.98)$ & $0.87(0.84 ; 0.91)$ \\
\hline \multirow[t]{4}{*}{ PF OA } & AUC & & & & & \\
\hline & & $0.62(0.59 ; 0.65)$ & $0.64(0.61 ; 0.67)$ & $0.65(0.63 ; 0.68)$ & $0.69(0.65 ; 0.73)$ & $0.68(0.64 ; 0.73)$ \\
\hline & Sons & $0.00(0.00 ; 0.00)$ & $0.22(0.17 ; 0.26)$ & $0.03(0.01 ; 0.06)$ & $0.00(0.00 ; 0.00)$ & $0.00(0.00 ; 0.00)$ \\
\hline & & $1.00(1.00 ; 1.00)$ & $0.93(0.91 ; 0.94)$ & $0.99(0.99 ; 1.00)$ & $1.00(1.00 ; 1.00)$ & $1.00(1.00 ; 1.00)$ \\
\hline
\end{tabular}

Conclusions: Neither MRI-defined inflammation, nor synovial/serum inflammation biomarkers at 2 years as analysed here predicted ROA or MROA at 5 years. The concordance between MRI and synovial inflammatory biomarkers was weak. Additional studies with longer follow-up will be needed to confirm or refute our find ings and to more firmly define the role of inflammation for OA development following acute ACL injury.

REFERENCE:

[1] Struglics A, et al. Changes in cytokines and aggrecan ARGS neoepitope in synovial fluid and serum and in C-terminal crosslinking telopeptide of type II collagen and $\mathrm{N}$-terminal crosslinking telopeptide of type I collagen in urine over five years after anterior cruciate ligament rupture: An exploratory analysis in the knee anterior cruciate ligament, nonsurgical versus surgical treatment trial. Arthritis Rheumatol 2015;67:1816-25.

Acknowledgements: We thank the participants of the KANON study without this work would not have been possible.

Disclosure of Interest: F. Roemer Shareholder of: Boston Imaging Core Lab (BICL), LLC., M. Englund: None declared, A. Turkiewicz: None declared, A. Struglics: None declared, A. Guermazi Shareholder of: Boston Imaging Core Lab (BICL), LLC., Consultant for: Sanofi-Aventis, Merck Serono, OrthoTrophix, AstraZeneca, Pfizer, GE, and TissuGene, L. S. Lohmander: None declared, S. Larsson: None declared, R. Frobell: None declared DOI: 10.1136/annrheumdis-2018-eular.2060

\section{OP0057 \\ EFFICACY AND SAFETY OF A DISTRACTION-ROTATION KNEE BRACE (ODRA) IN MEDIAL KNEE OSTEOARTHRITIS - A PHASE III RANDOMISED CONTROLLED TRIAL (ERGONOMIE STUDY)}

A. Diaz ${ }^{1}$, C. Morisset $^{2}$, I. Fournel ${ }^{3}$, A.-L. Soilly ${ }^{4}$, C. Bussiere ${ }^{5}$, A. Cherasse ${ }^{6}$, T. Conrozier ${ }^{7}$, D. Loeuille ${ }^{8}$, M. Timsit ${ }^{9}$, D. Wendling ${ }^{10}$, J.-M. Casillas ${ }^{11}$, J.F. Maillefert ${ }^{1}$, C. Binquet ${ }^{3}, \mathrm{P}$. Ornetti ${ }^{1}{ }^{1}{ }^{1}$ rheumatology, $\mathrm{CHU}$ dijon; ${ }^{2}$ physical medicine rehabilitation, PIT, CIC-P, ${ }^{3}$ epidemiology, $C I C, C H U$ DIJON; ${ }^{4}$ epidemiology, CHU Dijon, DRCI, dijon; ${ }^{5}$ orthopaedic surgery, dracy orthopaedic center, dracy; ${ }^{6}$ rheumatology, $\mathrm{CH}$ Macon, macon; ${ }^{7}$ rheumatology, $\mathrm{CH}$ Belfort, belfort, ${ }^{8}$ rheumatology, CHU nancy, nancy; ${ }^{9}$ physical medicine rehabilitation, Clinique Provence Bourbonne, aubagne; ${ }^{10}$ rheumatology, chu besancon, besancon; ${ }^{11}$ physical medicine Rehabilitation, $\mathrm{CHU}$ dijon, dijon, France

Background: According to EULAR and OARSI guidelines, evidence is inconclusive for the symptomatic benefits of an unloader knee brace in medial knee osteoarthritis (OA).

Objectives: The objective of this multicenter randomised controlled trial (RCT) (ClinicalTrials Id. NCT02765685) was to compare the efficacy and safety of the ODRA brace, a distraction-rotation custom-made knee brace versus usual care over one year in medial knee OA.

Methods: Patients with symptomatic medial knee OA (VAS pain scores at rest $>40 / 100$ for the medial compartment) and Kellgren-Lawrence (KL) grade II-IV were randomised in two groups: brace group (ODRA +usual care) vs usual care alone (UCA). Patients were followed up every two months for one year. Usua care consisted of all the pharmacological and non-pharmacological treatments used for the management of knee OA. The primary end-point was the difference of VAS-pain between M0 and M12. Secondary end-points included patient global assessment of disease severity (PGA) on VAS,\% of patients reaching the PASS (VAS-pain <30/100) and MCII (delta VAS-pain >20/100) thresholds at M12, KOOS scores, OA-specific quality of life questionnaire (OAKHQOL), as well as drug intake. Safety and compliance were evaluated by recording side effects and average knee brace duration of wear, respectively.

Results: Overall 120 patients (57\% women) from 7 centres were included. Their characteristics were the following: mean age $63.6 \pm 11.4$ years; BMI $29.6 \pm 5.5 \mathrm{~kg} /$ $\mathrm{m}^{2}$; OA duration $5.8 \pm 6$ years; $52 \% \mathrm{KL} \mathrm{III;} 21 \% \mathrm{KL}$ IV. The VAS pain decrease was statistically higher in the ODRA group (from $61.9 \pm 17.4(\mathrm{M0})$ to $38.8 \pm 25.3$ (M12) than in the UCA group $(54.9 \pm 18.2$ to $45.5 \pm 23.8)$ in the intent to treat bivariate and multivariate analyses (delta VAS-pain: 13.1 $\pm 4.9, p<0.01$ ). At M12, ODRA patients experienced a significant higher improvement than the UCA patients for PGA, all KOOS domains and for 3 of 5 domains of OAKHQOL (Pain, Physical activities, Mental health). Patients reaching PASS threshold at $\mathrm{M} 12$ were $42 \%$ in ODRA group vs $27 \%$ in UCA group (OR=3.04; $95 \% \mathrm{Cl}: 1.11$ to $8.30 ; \mathrm{p}<0.05)$ and were $46 \%$ vs $28 \%$ for $\mathrm{MCI}$ respectively (OR=2.65, $95 \% \mathrm{Cl}: 1.01$ to $6.96 ; \mathrm{p}<0.05)$. The overall analgesic use decreased more frequently in the ODRA group at M12 than in the UCA group ( $32.7 \%$ vs $15.4 \%, \mathrm{p}<0.05)$ except for NSAIDs, HA or steroid injections. The compliance was good: the brace was worn for a median [IQR] duration of 5.7 [4.0-7.0] hours a day between $\mathrm{M} 0$ and $\mathrm{M} 6$ and 5.3 [3.7-6.3] hours a day between M6 and M12. Non-serious side effects were more common in the ODRA group $(p<0.05)$ which justified the definitive withdrawal of the brace in 8 ODRA patients (13\%), mainly for cutaneous side effects.

Conclusions: In this RCT, the use of the ODRA brace in addition to usual care was shown to be superior to usual care alone in reducing symptoms and improving quality of life of patients suffering from medial knee OA, with good compliance.

Disclosure of Interest: None declared

DOI: 10.1136/annrheumdis-2018-eular.4927

\section{OP0058 LOW-DOSE RADIATION THERAPY AS TREATMENT FOR HAND AND KNEE OSTEOARTHRITIS: TWO DOUBLE BLINDED RCT'S}

M. Minten ${ }^{1}$, E. Mahler ${ }^{1}$, M. Leseman-Hoogenboom ${ }^{2}$, S. Boks ${ }^{3}$, M. Kloppenburg ${ }^{4}$, J. W. Leer ${ }^{2}$, P. Poortmans ${ }^{5}$, F. van den Hoogen ${ }^{1,6}$, A. den Broeder ${ }^{1,6}$, C. van den Ende ${ }^{1,6} .{ }^{1}$ Rheumatology, Sint Maartenskliniek; ${ }^{2}$ Radiation Oncology, Radboud university medical centre; ${ }^{3}$ Radiology, Sint Maartenskliniek, Nijmegen;

${ }^{4}$ Rheumatology, Leiden university medical centre, Leiden, Netherlands; ${ }^{5}$ Radiation Oncology, Institut Curie, Paris, France; ${ }^{6}$ Rheumatology, Radboud university medical centre, Nijmegen, Netherlands

Background: Synovial inflammation plays an important role in osteoarthritis (OA) pathophysiology. In some countries, low-dose radiation therapy (LD-RT) is widely used as treatment for OA, while relatively unknown in others. Studies in vitro and in OA animal models have shown anti-inflammatory effects of LD-RT. However systematic literature review has shown that high-level evidence for beneficial effects in clinical practice is lacking.

Objectives: To assess the effect of LD-RT on clinical outcomes and inflammation in patients with hand or knee OA, using two parallel prospective RCTs. 